\title{
Construction of Signal Processing Course Group for Electrical Information Major Disciplines
}

\author{
Yu Shang \\ School of Electronics and Information Engineering \\ Xi'an Technological University \\ Xi'an 710021, Shanxi, China \\ 179648622@qq.com
}

\author{
Mengqiao Lu \\ School of Electronics and Information Engineering \\ Xi'an Technological University \\ Xi'an 710021, Shanxi, China \\ 353729958@qq.com
}

\begin{abstract}
Signal processing courses are the important basic courses of electronic information major. With the development of information technology, the application of signal processing has become much more widely. Nearly all the electricity-related majors offer such courses. However, the connotation of such courses in many majors is not clear, leading to some problems in the setting of the courses. This article mainly elaborates the main research content of the series of signal processing courses, analyzes the relationship between courses, courses and related courses within the curriculum system, and designs two new kinds of curriculum system maps for different majors and the courses within the curriculum group. The overall teaching contents are optimized and experiment system designed based on CDIO. Through the teaching practice, the whole teaching content of the course group is more reasonable, and the experiment content designed based on CDIO has a good effect, which effectively improves the teaching effect of the signal processing courses.
\end{abstract}

Keywords-Signal processing; Curriculum system; Optimization; Experiment system

\section{INTRODUCTION}

In recent years, with the development of artificial intelligence technology with core technologies such as language processing, image processing, and big data analysis and processing, the technology for acquiring and processing digital signals has expanded from manufacturing to agriculture, medical care, education, energy, and national defense, and brought more development to the human society. As a result, with the expansion of the scope of signal processing theory and method applications, the series of signal processing courses have become the core courses for various engineering majors around the world. In addition to the electronics majors, optoelectronics, electro mechanics, computers, and biomedical sciences have also set up series of signal processing courses. Because of the convergence between the original curriculum system and signal processing courses, there are various problems in the construction of teaching system and the arrangement of teaching content.

\section{The Curriculum Group Construction IDEAS}

The establishment of the curriculum system should face the development of worldwide higher education, update the concept of engineering education, explore and practice new teaching thoughts, based on signal processing courses, and broaden the electrical specialty knowledge module as a breakthrough. We should reconstruct signal processing courses system. Scientific research and higher engineering education research should be timely introduced to strengthen basic skills and the overall quality training, which will realize the educational objective of a broad range of subjects with universality and establish a good mechanism for sustainable development.

Since the series of signal processing courses all use mathematics as the main tool, their theoretical and practical applications are very strong. There is a certain degree of overlap between the courses within the system and related courses, and the content of each course needs to be analyzed. Focus on clarifying the interrelationships between courses to ensure the effective convergence of curriculum content, optimize and integrate the curriculum as a whole, a modular theory teaching system is formed and a layered practical teaching system on major knowledge platforms is established. Under this system, we pay attention to the organic integration of theory and experimental teaching, and pay attention to the cultivation of engineering application ability [1].

\section{COURSE SySTEM CONSTRUCTION}

\section{A. Build Maps of Basic Courses}

Through in-depth study of the knowledge related to the pre-opening and follow-up courses of the signal processing series in each major, and in accordance with the requirements of the "National Program of Outstanding Engineers", the knowledge matrix is constructed according to knowledge areas and knowledge points, and the cohesion between various knowledge points is fluent. To further clarify the corresponding courses for the semester, so as to make it more reasonable in the training plan to lay the foundation.

Among the major subjects of electrical information, through the core curriculum of education, the signal processing courses mainly include two core courses: "Signal and System", and "Digital Signal Processing”. According to professional requirements, there are other core courses such as "Signal Analysis and Processing", "DSP technology and applications”, and series of experimental courses [2]. According to the course of each major, the Map of the basic curriculum system of electrical and electronic is constructed, as shown in Fig. 1, which can be used for professional reference. The major that requires a higher level of signal processing can adopt (a), and the lower major can adopt (b) 
graph structure. Each major can choose the course and the

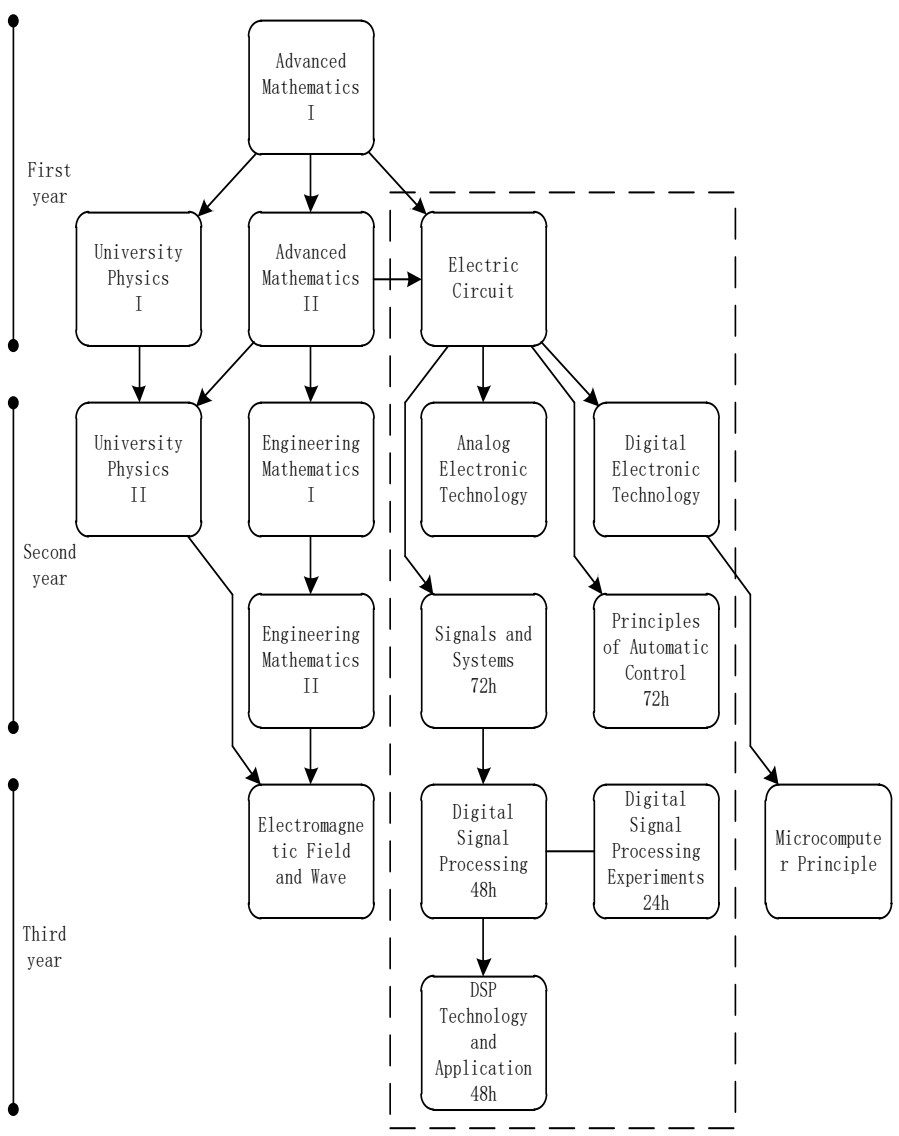

$(\bar{a})$ term study according to the actual need.

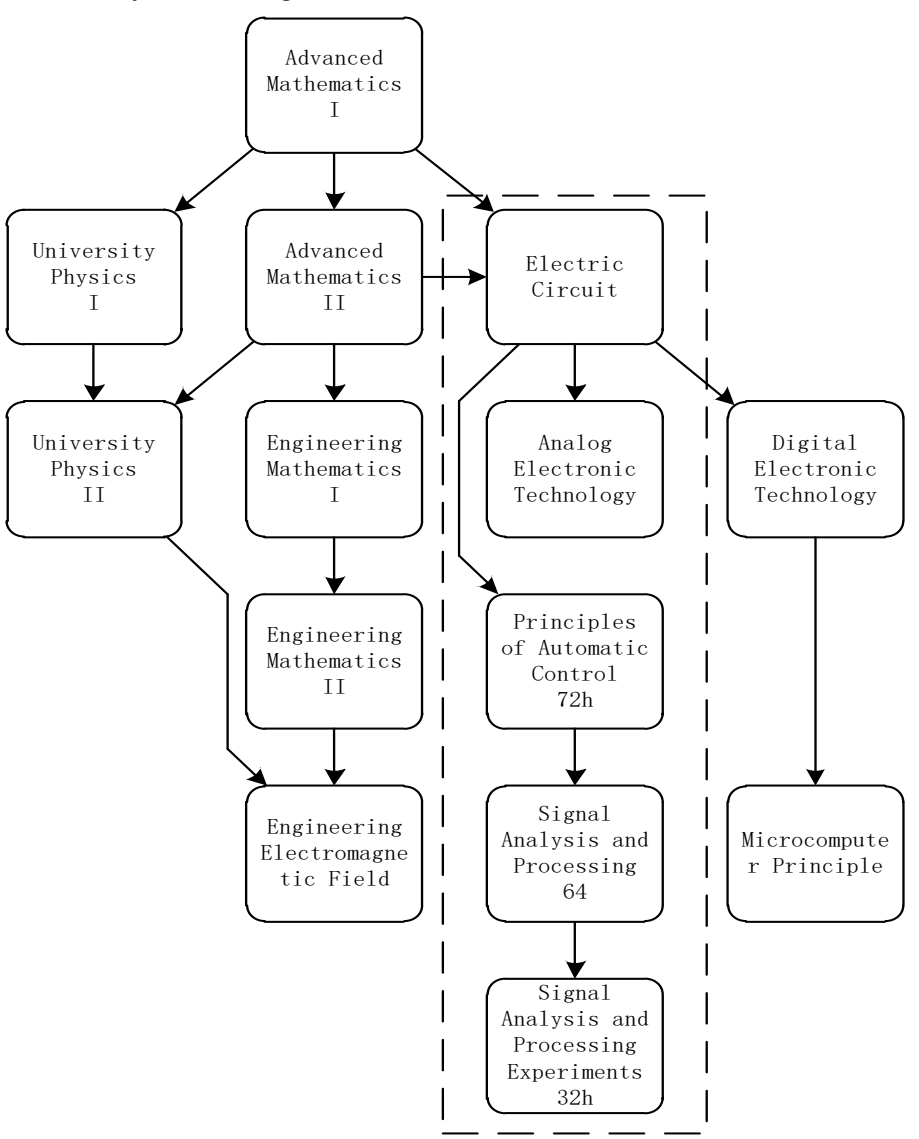

(b)

Fig. 1. Map of basic curriculum system for Electrical and Electronic

The basic course of the electrical major is "Circuit" (nonelectrical specialty is the "Circuit Analysis") course, it is also the theoretical foundation of "Principle of Automatic Control " and signal processing series course. The basic course "Signal and System" in the general course group should be set in the semester after the "Circuit". The "Principle of Automatic Control" course could generally be set up simultaneously with "Signals and Systems". If some majors do not require high levels of signal processing professional courses, it is recommended to set up "Signal Analysis and Processing". The recommended hours of "credit" is 64 (at least 48). The teaching experience is well established after the "Principle of Automatic Control".

\section{B. Establish a Curriculum Group Architecture}

The knowledge and ability matrixes of the courses in the course group is constructed to realize an organic combination of principle, method and application, in accordance with Signal Analysis $\rightarrow$ System Analysis $\rightarrow$ Digital Processing of Signals $\rightarrow$ Realization of the System structural order. Each major can choose the teaching contents and hours to adapt to itself to form a well-worked teaching system with complete teaching content and reasonable division. The structure of signal processing course group is established, as shown in Fig. 2.

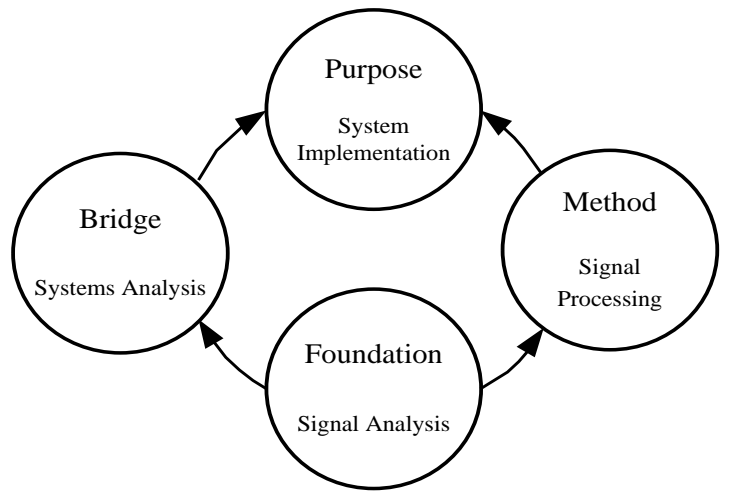

Fig. 2. Structure of signal processing course group

In the course group, the core basis are the two courses of "Signal and System" and "Digital Signal Processing". The foundation is the analysis and processing of the continuous time signal and system, while the purpose is the analysis and processing of the discrete time signal and system. The core concept of signal processing should be the analysis and processing of frequency domain in discrete domain. 


\section{The Overall Optimization Design of Curriculum Group TEACHING CONTENT}

\section{A. "Signals and Systems"}

The traditional content of "Signal and System" includes two parts: signal analysis and linear system analysis. According to the time domain of the object of study, there are two kinds of systems: continuous time and discrete time signal and system. According to the processing method, it can be divided into two kinds: time domain analysis method and transform domain analysis method. The transform domain method is mainly summarized as frequency domain, complex frequency domain and $\mathrm{Z}$ transform. The most important operation is convolution integral or convolution sum. Today, due to the popularity of computer technology and various engineering software, from the perspective of transform domain, the emphasis of the course should be on signal spectrum analysis and system from the point of view of time domain, the emphasis of the course should be on discrete domain analysis. The solution of the time-domain equation of the circuit should be weakened, while the solution of the inverse transformation should be weakened.

\section{B. "Circuit Theory" and "Signals and Systems"}

The "Circuit" should mainly introduce the basic concept and the circuit law. Its linear resistance circuit, the method and theorem of the time domain analysis of the linear dynamic circuit are the foundation of all the subsequent courses. All can be properly reduced except the major of Electrical Engineering. Fourier series, complex frequency domain analysis and so on can be put into the subsequent "Signal and Systems" course.

\section{C. "Signals and Systems" and "Principle of Automatic Control"}

These two courses are the basic core courses of weak electricity and strong electricity respectively. The main contents include the analysis of linear system. All the transform fields are involved except for the frequency field, and the content is inevitably overlapped. 'Principle of Automatic Control” focuses on the analysis of the system, masters the basic principles of the control system and analysis methods such as the stability and controllability of the control system, and pays attention to the modeling of the system analysis and synthesis. "Signal and System" pays more attention to signal analysis, especially signal frequency domain analysis, from the spectrum analysis point of view between the signal and the system relationship [2-5].

\section{D. "Signals and Systems" and "Digital Signal Processing"}

"Signals and Systems" is the theoretical basis of "Digital Signal Processing". In terms of theory, both are the results of the same theoretical system after the breakdown, and there is no repetition of the content itself. In order to ensure the integrity of the teaching content, the sampling theorem, discrete signal and system, content analysis method in frequency domain are expounded again in most of the digital signal processing system and teaching materials.

The teaching contents of "Digital Signal Processing" focus on the engineering practice, and highlight the digitization methods and techniques. The emphasis should be placed on the DFT,FFT algorithm, the principle and design method of the digital filter, and the discrete time Fourier transform. The basic concepts such as discrete Fourier series, can be shifted downwards, and the contents of modern signal processing added appropriately [2-5].

\section{E. "Signal Analysis and Processing"}

The course "Signal Analysis and Processing" is a combination of "Signal and System" and 'Digital Signal Processing". If "Principle of Automatic Control "is offered in a major, it will be suggested as a subsequent course. Due to the limited study hours, the course generally can only involves the basic principles and methods of signal analysis and processing: Fourier spectrum of signal, sampling theorem, and so on. Signal transmission and filtering, DTFT,DFT,FFT, digital filter basic design method and so on.

\section{EXPERIMENTAL COURSE SySTEM CONSTRUCTION}

\section{A. Hierarchical design practice teaching system}

According to "signal analysis $\rightarrow$ system analysis $\rightarrow$ digital signal processing $\rightarrow$ system realization", the entire curriculum group experiment system content is organized, experimental methods are reformed, basic experiments are emphasized, and comprehensive and design experiments are added. Its hierarchical structure is shown in Fig. 3. [6].

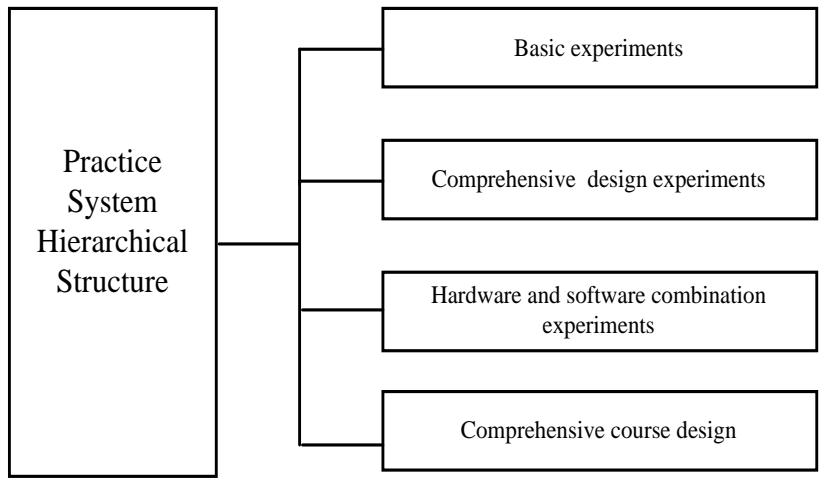

Fig. 3. Practice System Hierarchy

With the development of signal processing disciplines, the emergence of mass storage has made it easier to implement in DSP with C language and other high-level languages, so that we can be closer to the actual engineering development process in the laboratory. The design process of signal processing is realized by a combination of software and hardware. Based on the overall planning of experimental teaching content in the signal processing curriculum system, different majors can adopt different implementation schemes. In the experimental design of the entire series of courses, we carried out experimental designs according to the corresponding relationship between the basic software simulation experiment based on the MATLAB software platform and DSP or ARM hardware implementation, and constructed an integrated and design-based experiment based on the actual system. Let the experiment be closer to the actual digital signal processing system. 
B. Design experimental content based on the principles of the CDIO concept"

The actual digital signal processing system such as ECG signal processing and voice signal processing, in scientific research projects is planned as the first-level project content. From the view of engineering application, the second and third level items are designed. As shown in Fig. 4, the experimental content has a progressive hierarchy.

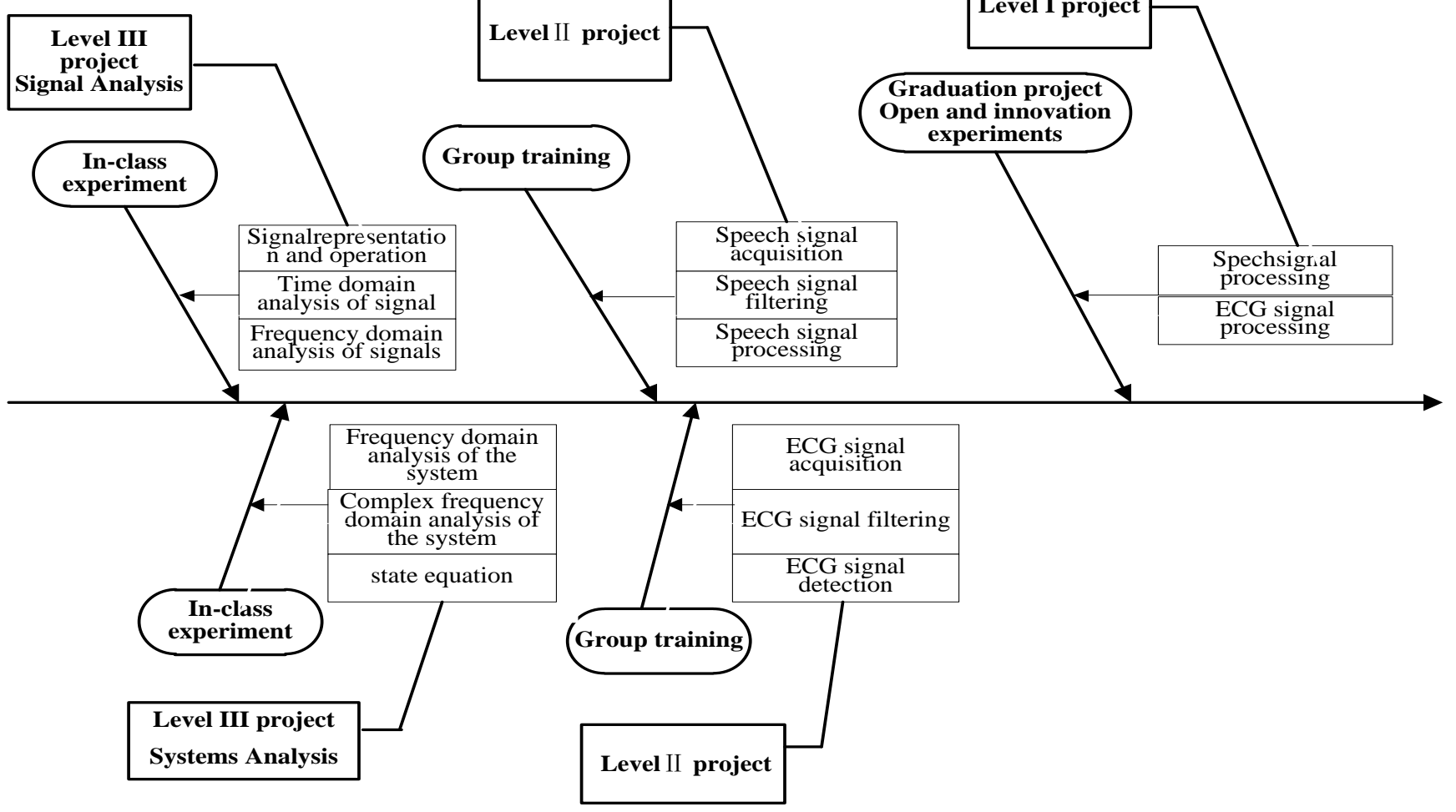

Fig. 4. Structure of Signal Analysis and Processing Experiment System

Basic experiments mainly include fundamental signal operations, implementation of typical signals, convolution and related implementation, spectrum analysis of continuous signals, frequency spectrum analysis of discrete signals, time and frequency domain sampling, system analysis, etc.. The comprehensive design experiments are mainly a series of filtering experiments constructed by the subsystems of the signal processing system, and the software and hardware combination experiments and the integrated curriculum designs mainly focus on the frequency spectrum and filter processing of signals of FFT analysis, signal sampling and filtering, and DSP/ARM implementation of speech, ECG signal compression, real-time filtering, spectrum analysis and so on.

\section{C. "Exploration of new practice teaching methods"}

In the implementation, we try to use it as a large-scale program design assignment to cultivate the students' engineering innovation and team cooperation consciousness. The specific steps are as follows:

1) Developed a second-level project-driven program design task. The task book only provides the engineering project background and the required design index. The specific design proposal is not provided. The signal that the design needs to be processed is also collected by the students themselves.

2) Implementation time: mid-term to end of the course.

3) Implementation: The students are appointed to set up 3member groups and divided labor freely. In order to get closer to the actual work, individuals can not choose partnership which can train students' ability to cooperate.

4) Project assessment: The results should be assessed by writing a design report. Students need to submit source programs and design reports. The project team writes the project report in accordance with the specifications, and submits the report, relevant data, source code and operating results within a specified time, and analyzes the results. When students submit their reports, they submit their own selfevaluation results and mutual assessment results at the same time. The final student's personal score is weighted by the project group's score and the self-evaluation's score.

Judging from the implementation effect, students completed the design task on time with unexpected enthusiasm, and made many constructive suggestions during the implementation process, which received good results. 


\section{SUMMARY}

With the development of technology, the content of the course system is constantly updated. It is necessary for us to study and explore how to construct the signal processing course system in accordance with the requirements of electrical information, and optimize teaching and practical teaching. Through the research and construction of signal processing theory and practical teaching system, we hope that students can be familiar with theoretical knowledge, master system design methods and basic skills from software simulation to hardware processing, and improve their ability to find problems, raise and solve problems in practice.

\section{REFERENCES}

[1] Wang Yanfen, Zhang Xiaoguang, Wang Gang, et al. Construction and Reform of Signal Processing Courses for Electronics and Information Specialty [J]. Experimental Technology and Management, 2015, pp. 1114. (In Chinese)

[2] Nie WY, Wang Zhonggen, Liao Xiaowei. Discussion on Optimization of Circuit Analysis, signal and system, and Digital signal processing. Science \& Technology Information, No. 5, 2012, pp. 24-25. (In Chinese)

[3] Liu Yongxiang, Wu Jing, Li Xiang. Research on Signal and System Course Teaching Model for International First-rate Universities [J]. Journal of Higher Education Research, 2011, pp. 77-79. (In Chinese)

[4] Bu Fangling, Xu Xin, Zou Lian, et al. Reform of Signal and System Teaching for Innovation Ability Cultivation [J]. Computer Education, 2016, pp. 52-55. (In Chinese)

[5] Liu Cui-xiang et al. Research on practical Teaching of signal processing courses for Electronic Information Specialty. China Electric Power Teaching, No. 5, 2014, pp. 170-171.(In Chinese)

[6] Chen Liwei. Research and Construction of signal processing practice Teaching system for the New Talent training Program. Creative Education, No. 5, 2013, pp. 55-56. (In Chinese) 